\title{
Escala de Deseabilidad Social: Análisis psicométrico en muestra argentina.
}

\author{
María José Pérez *1, Mabel Labiano ** \& Claudia Brusasca *** \\ * Lic. en Psicología. Docente Área Psicobiológica Departamento de Psicología. \\ **Dra. en Psicología. Profesora Titular Exclusiva en la Facultad de Química, Bioquímica y Farmacia. Directora \\ del PROICO 22H915 de Ciencia y Técnica. \\ ***Mag. en Neuropsicología. Profesora Adjunta Exclusiva. Integrante del PROICO 22H915 de Ciencia y \\ Técnica. \\ Facultad de Ciencias Humanas de la Universidad Nacional de San Luis. San Luis, Argentina.
}

\begin{abstract}
Resumen: La Deseabilidad Social (DS) es la tendencia psicológica a auto-atribuirse cualidades de personalidad socialmente deseables y rechazar aquellas indeseables. Se estudiaron las propiedades psicométricas de la Escala de DS de Marlowe y Crowne (M-C) en una muestra de 400 sujetos pertenecientes a provincias cuyanas de Argentina (rango de edad: 17-70 años; $\mathrm{M}=30.97 ; \mathrm{DE}=12.97$ ). Se indagó la consistencia interna del test; capacidad discriminativa de los ítems; se establecieron correlaciones entre variables sociodemográficas (edad, escolaridad, sexo) y puntajes de DS; comparándose los resultados encontrados con los de otros investigadores. Se observó que a mayor edad disminuía la tendencia a mostrar una imagen socialmente aceptable. Se obtuvo una versión reducida del instrumento original, con una estructura factorial bidimensional y una consistencia interna que se incrementó de .48 a .57. Se concluye que dicha escala sería un instrumento moderadamente fiable para su aplicación en la población cuyana.
\end{abstract}

Palabras clave: Deseabilidad Social; Escala de Deseabilidad Social; Confiabilidad; Validez.

Title: Desirability Scale: Psicometric analysis in Argentinean sample.

Abstract: Social Desirability (SD) is defined as the psychological tendency to self-attribute personality qualities socially desirable as well as to reject those supposedly undesirable. Psychometric properties of Marlowe-Crowne SD Scale (1960) were studied in a sample of 400 individuals (age rank: 17-7; M=30.97; DE=12.97) of Cuyo popuation (Argentinian people). The inner consistence of test; the discriminative capacity of items was investigated. Also, correlations between sociodemographic variables as age, instruction level, sex and scores were obtaneid. The results finded were compared with the results of others researchers. Correlations between several socio-demographic variables were established and it was observed that as the age increased the tendency to show a socially accepted image decreased. A version reduced of original instrument, with a factorial bidimensional structure was obtaneid, and a inner consistence incremented of .48 to .57 . It is concluded that SD scale maybe a moderately reliable instrument applicable to Cuyo population.

Key Words: Social Desirability; Social Desirability Scale; Reliability; Validity.

\section{Introducción}

Es bien conocido que los tests de personalidad muestran, en general, mayores dificultades psicométricas que los tests de capacidad o de rendimiento máximo. Uno de los cuestionarios que ha recibido mayor atención ha sido el de DS de Marlowe y Crowne (1960).

Algunos autores afirman que la DS no es un rasgo unitario sino multidimensional (Pauhlus, 1984). Eysenck y Eysenck (1976) sugieren que las escalas de DS miden un rasgo estable cuando se administran en condiciones neutrales o de baja motivación, pasando a

\footnotetext{
${ }^{1}$ Por favor dirigir la correspondencia relacionada con este artículo a:

María José Pérez, E-mail: mjperez@unsl.edu.ar-majo_111@live.com.ar.

Dirección Postal: UNSL. Ejército de los Andes 950. (C.P. 5700). San Luis. Argentina. Teléfono: (02652) 15211406
} 
medir disimulación en situaciones de presión. Edwards (1957) describió a la DS como la medición de "la tendencia a dar respuestas socialmente deseables de autodescripción", más concretamente, una característica individual de autopresentación sin instrucciones especiales o motivación para hacerlo.

Sin embargo, el tema no es tan simple y tampoco está claro cual es la validez predictiva de las puntuaciones en DS considerada como rasgo de personalidad. También se ha señalado que el cuestionario resultaría útil para predecir rasgos psicopatológicos.

La necesidad de disponer de cuestionarios de rasgos de personalidad (específicamente de DS) adaptados a nuestra población argentina (región de Cuyo), y la carencia de los mismos, dio fundamento al presente trabajo de investigación.

El sesgo de respuesta es un tema preocupante en la evaluación psicológica, particularmente en las encuestas de autoevaluación. El más estudiado es la Respuesta Socialmente Deseable (RSD).

Hace más de 50 años los investigadores en psicometría ya habían planteado la cuestión de los efectos de la RSD sobre la validez de los cuestionarios (Bernreuter, 1933; Vernon, 1934). Diez años más tarde, Meehl y Hathaway (1946) fueron capaces de citar ocho mediciones específicamente desarrolladas para indexar las RSD en las autoevaluaciones. De este modo, dichos autores construyeron en 1946, la Escala MMPI (Mentira) que contiene 15 declaraciones acerca de las actitudes y prácticas que son socialmente indeseables pero comunes. Marcar "falso" en ocho o más ítems se considera evidencia de que el encuestado es bueno fingiendo en el MMPI. La medida se corresponde con el segundo factor de RSD, altamente correlacionada con otras escalas de mentira y la Escala de M-C. También Meehl y Hathaway desarrollaron la Escala MMPI K, que consta de 30 ítems diseñados para identificar personas anormales cuyas puntuaciones MMPI parecen normales. La Escala MMPI L (desarrollada por los mismos autores en 1946), es una medición más sutil de RSD.

Desde la década de 1950, la RSD ha sido una preocupación destacada en la medición de la personalidad:

En 1952, Gough desarrolló una Escala CPI Buena Impresión, que fue diseñada para medir lo que la gente dice de sí misma cuando tratan de crear una impresión altamente valorable en los otros. Esta escala fue publicada en 1960 y utilizada como instrumento de medición en autoinformes. Marlowe y Crowne (1964) descubrieron una amplia gama de correlación que sugiere la existencia de una construcción subyacente, a saber, la necesidad de 
aprobación.

En 1965, Crandall, Crandall y Katkovsky construyeron una Escala de DS Infantil, diseñada para niños de entre 6 y 12 años. El contenido de los ítems es bastante similar al contenido de la escala de M-C, pero está redactado en lenguaje infantil.

En 1978, Schuessler, Hittle y Cardascia desarrollaron la Respuesta Deseable en Actitudes y Opiniones, diseñada específicamente para detectar RSD en encuestas de actitudes y opiniones de la población en general. Un impresionante conjunto de normas está disponible a partir de una muestra de probabilidad nacional. Los ítems fueron monitoreados para evitar diferencias entre sub-grupos, considerando variables tales como la raza y la educación.

Paulhus, en 1984, construyó el Inventario Equilibrado de Respuesta Deseable (BIDR), que contiene distintas medidas de gestión de impresiones (público impulsado por la libre expresión) y auto-mejora engañosa (un honesto sesgo de positividad de sí mismo). La suma de las dos mediciones se correlaciona altamente con la Escala M-C.

El análisis de los instrumentos de RSD ha revelado dos factores primarios (Borkenau \& Ostendorf, 1989; Paulhus, 1984; Edwards \& Walsh, 1964; Wiggins, 1964; Jackson \& Messick, 1962). Un grupo se asocia a Alpha, el factor de ansiedad general de la MMPI (Block, 1965); el otro factor llamado Gamma (Wiggins, 1964) está vinculado a lo agradable y al tradicionalismo. Paulhus $(1984,1986)$ presentó pruebas de que estos dos factores de RSD representan: (a) respuesta libre de positividad engañosa o "positividad de autoengaño" (una honesta pero demasiado positiva presentación de sí mismo), y (b) la gestión de la impresión (auto-presentación adaptada a un público). La escala M-C carga sobre ambos factores, aunque más en la gestión de impresión (Paulhus, 1984).

El término "gestión de impresión" fue elegido para representar el punto de vista tradicional de la RSD, algunas veces con el propósito de adaptar las respuestas para crear una imagen más positiva de sí mismo. De las muchas impresiones que uno puede tratar en la actualidad, este factor representa sólo una: una persona socialmente convencional y dependiente. El rótulo “impresión de la gestión” es preferible a “mentir", una acusación demasiado dura. Después de todo, algunas personas pueden mentirse a sí mismas sólo para evitar la desaprobación social (Crowne, 1979). Cualquiera sea la etiqueta, esta tendencia variará en función de la situación demandada y motivos transitorios, y tal variación puede “oscurecer" la validez de los informes de los entrevistados.

Por lo tanto, resultó de gran interés en la presente investigación analizar la 
consistencia interna de la Escala M-C para adaptarla a nuestra población en particular, dado que es considerado el instrumento más popular y utilizado para medir el rasgo de DS (Reynolds, 1992; Schmitt \& Steyer, 1993). La importancia de su utilización en la disciplina psicológica radica, entre otros aspectos, en que rasgos acentuados de DS pueden sesgar los resultados de investigaciones científicas.

Este trabajo se orientó a explorar las propiedades psicométricas del cuestionario de DS de M-C (1960) en una muestra poblacional de la zona de Cuyo (Argentina).

Como objetivos específicos se plantearon los siguientes: analizar la capacidad discriminativa de los ítems; extraer los componentes principales, obteniendo la matriz factorial del cuestionario; explorar la consistencia interna del cuestionario; establecer correlaciones entre variables sociodemográficas (edad, escolaridad, sexo) y los puntajes de DS; obteniendo una versión reducida del cuestionario aplicable a la población estudiada.

\section{Método}

\subsection{Participantes.}

Se aplicó el instrumento a un total de 400 sujetos de ambos sexos (245 mujeres y 155 hombres), de diferentes condiciones socioeconómicas; con un rango de edad comprendido entre 17 y 70 años $(M=30.97 ; D E=12.97)$. La totalidad de los sujetos pertenecían a provincias cuyanas (San Luis, Mendoza y San Juan) de Argentina. La participación fue voluntaria y con previo consentimiento informado. Más del $50 \%$ de los participantes fueron mujeres, jóvenes y solteras. Una proporción importante de la muestra estuvo constituida por estudiantes universitarios (casi un 50\%); en segundo término, por empleados de instituciones estatales o privadas ( 88 sujetos).

\subsection{Instrumento.}

Escala de Deseabilidad Social (Marlowe y Crowne, 1960): Intenta medir la tendencia a dar una imagen favorable de sí mismo, ante diversas situaciones propuestas. Originalmente, está compuesta por 33 ítems, con un formato de respuesta dicotómico (verdadero-falso) y presenta diferentes situaciones de la vida cotidiana ante las cuales el sujeto debe responder con una de las dos opciones posibles.

\subsection{Procedimiento.}

El cuestionario definitivo fue aplicado en condiciones semejantes a todos los sujetos 
de la muestra, obteniéndose los datos sociodemográficos correspondientes. Se garantizó el anonimato de los resultados individuales. Para el procesamiento de datos se utilizó el software de análisis estadístico SPSS versión 15.0.

En una primera etapa, se llevó a cabo el análisis de los reactivos y la modificación y adaptación de los mismos a un lenguaje familiar, accesible y claro. Previamente, los reactivos fueron sometidos a juicios de expertos en psicología, realizándose una aplicación piloto a una muestra pequeña, antes de pasar a la aplicación del instrumento definitivo a la muestra mayor de la población cuyana.

En una segunda etapa, se llevó a cabo el análisis psicométrico de la escala original. Para cada ítem fue tabulada la información descriptiva y se identificaron los reactivos débiles para su eliminación, obteniéndose una versión reducida.

Se realizó un análisis factorial para evaluar la estructura interna de la escala. La adecuación muestral para el análisis factorial se evaluó con las pruebas de KMO y prueba de Bartlett. La selección del número de factores se hizo a partir del gráfico de sedimentación y las cargas factoriales fueron sometidas a rotación Varimax.

Para el análisis de confiabilidad, se utilizó el coeficiente Alfa de Cronbach del total de la escala así como de cada factor de la misma.

\section{Resultados}

Se Se observó que la distribución de la muestra estudiada fue adecuada para realizar un análisis factorial, ya que el índice KMO fue de .84 (ver Tabla 1).

Tabla 1.

Estructura interna de la escala de M-C original.

\begin{tabular}{lrr}
\hline Medida de adecuación muestral de Kaiser-Meyer-Olkin. & .84 \\
\hline Prueba de esfericidad de Bartlett & Chi-cuadrado aproximado & 2294.28 \\
& Gl & 528 \\
& Sig. & .000 \\
\hline
\end{tabular}

A continuación, el método de extracción de factores utilizado fue el de Análisis de Componentes Principales (ver Figura 1) el cual permite explicar la mayor cantidad de varianza posible de los datos obtenidos. Según el método del gráfico de sedimentación 
aplicado, se observó claramente que podían seleccionarse dos factores. Estos explicaron un $24.42 \%$ de la varianza.

Gráfico de sedimentación

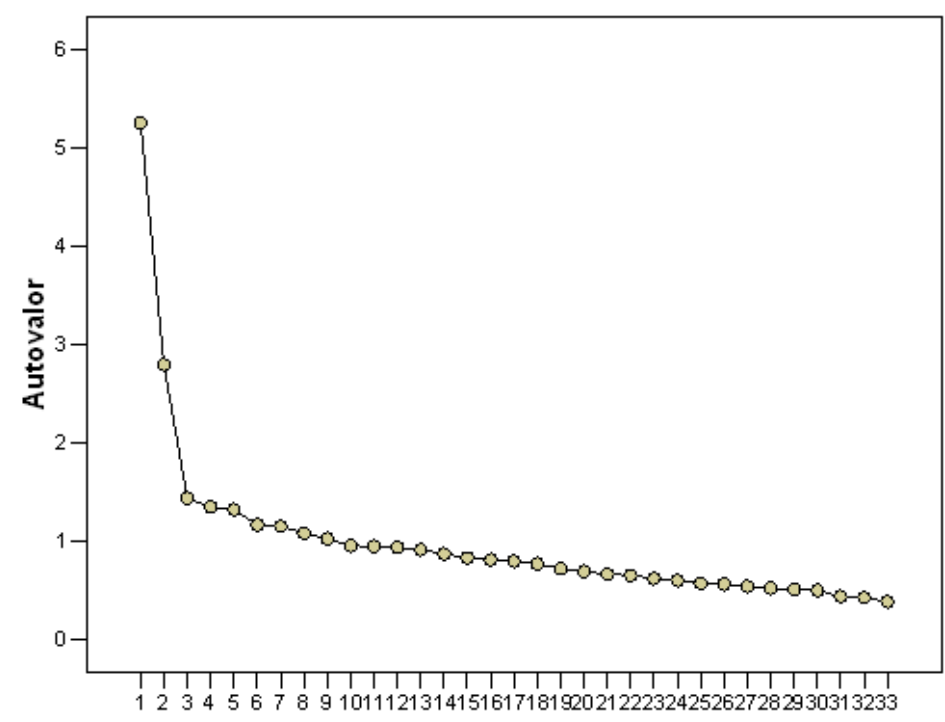

Número de componente

Figura 1. Análisis de componentes principales de la muestra total $(\mathrm{N}=400)$ en la escala Marlowe Crowne original.

Tabla 2.

Estadísticos total-elemento: correlación entre cada item y el total de la escala $M-C$.

Correlación elementototal ESCALA MARLOWE - CROWNE corregida

1) Antes de votar averiguo a fondo las capacidades de todos los candidatos .27

2) Nunca dudo en dejar mis cosas de lado para ayudar a otro que está en problemas

3) Algunas veces me es difícil continuar con mi trabajo si no estoy estimulado

4) Nunca tengo una antipatía intensa hacia alguien

5) 5) En ocasiones, he tenido dudas acerca de mi habilidad para tener éxito en la vida .20

6) Algunas veces me siento mal cuando no puedo encontrar mi camino .15

7) Siempre soy cuidadoso acerca de mi manera de vestir .23

8) Cuando como en casa, mis modales son tan adecuados como cuando como afuera en un restaurante .35 
ESCALA MARLOWE - CROWNE

8) Cuando como en casa, mis modales son tan adecuados como cuando como afuera en un restaurante

9) Si pudiera entrar a un cine sin pagar y estar seguro de no ser visto, probablemente lo haría

10) En pocas ocasiones he dejado de hacer algo debido a que me consideré poco capaz

11) A veces me gusta chusmear

12) A veces me he rebelado contra personas con autoridad, aunque sabía que estaban en lo cierto

13) No importa quien me está hablando, siempre soy un buen escucha

14) Alguna vez "me hice el enfermo" para evitar algo

15) En ciertas ocasiones tomé ventajas de alguien

16) Siempre estoy dispuesto a admitir cuando cometo un error

17) Siempre trato de practicar lo que predico

18) No es particularmente difícil para mí relacionarme con gente bocona, detestable

19) Algunas veces trato de vengarme más que perdonar y olvidar

20) Cuando no sé algo, no tengo problema en admitirlo

21) Siempre soy amable, aún con personas que son desagradables

22) Realmente, a veces he insistido en hacer cosas a mi manera

23) Ha habido ocasiones en las que me sentí como "quebrado"

24) Nunca permitiría que alguien sea castigado por mi culpa

25) Nunca me resiento si me piden que devuelva un favor

26) Nunca me he molestado cuando las personas expresan ideas muy diferentes a las mías

27) Nunca hago un viaje largo sin comprobar la seguridad de mi auto

28) A veces estuve muy envidioso de la buena suerte de los otros

29) Casi nunca he sentido el impulso de dejar de lado a alguien

30) Nunca he sentido que fui castigado sin motivo

31) Algunas veces pienso, cuando los otros tienen una desgracia, que ellos solo tienen lo que se merecen

32) Nunca he dicho deliberadamente algo que hiera los sentimientos de alguien

33) Algunas veces me ha molestado que la gente me pida favores
Correlación

elemento-

total

corregida 
En la Tabla 2 se indica la correlación encontrada entre cada reactivo y el total de la escala. A partir de los dos componentes extraídos, se utilizó el procedimiento de rotación, que permite representar los factores como ejes de referencia, así como observar la carga factorial de cada variable. La matriz de componentes rotados con el método Varimax, facilita la interpretación teórica de los factores hallados. Se aprecia (ver Tabla 3) que el ítem 28 (" $A$ veces estuve muy envidioso de la buena suerte de los otros") es un ítem complejo ya que no carga únicamente en un solo factor. Los ítems: 33 ("Algunas veces me ha molestado que la gente me pida favores"); 7 ("Siempre soy cuidadoso acerca de mi manera de vestir"); 31 (“Algunas veces pienso, cuando los otros tienen una desgracia, que ellos solo tienen lo que se merecen"); 29 ("Casi nunca he sentido el impulso de dejar de lado a alguien"); 1 ("Antes de votar averiguo a fondo las capacidades de todos los candidatos"); 4 ("Nunca tengo una antipatía intensa hacia alguien Nunca tengo una antipatía intensa hacia alguien"); 30 ("Casi nunca he sentido que fui castigado sin motivo") y 32 ("Nunca he dicho deliberadamente algo que hiera los sentimientos de alguien") son ítems con baja carga factorial, ya que su aporte es inferior a 0.40 .

\section{Tabla 3.}

Componentes rotados de la Escala Marlowe Crowne

\begin{tabular}{ll}
\hline & \multicolumn{2}{c}{ Componente } \\
ITEMS DE LA ESCALA MARLOWE-CROWNE & $\mathbf{1}$ \\
\hline 24. Nunca permitiría que alguien sea castigado por mi culpa & .62 \\
13. No importa quien me está hablando, siempre soy un buen escucha & .58 \\
20. Cuando no sé algo, no tengo problema en admitirlo & .58 \\
17. Siempre trato de practicar lo que predico & .57 \\
16. Siempre estoy dispuesto a admitir cuando cometo un error & .56 \\
25. Nunca me resiento si me piden que devuelva un favor & .56 \\
26. Nunca me he molestado cuando las personas expresan ideas muy diferentes a & .55 \\
las mías & .52 \\
15. En ciertas ocasiones tomé ventajas de alguien & .52 \\
19. Algunas veces trato de vengarme más que perdonar y olvidar & .49 \\
2. Nunca dudo en dejar mis cosas de lado para ayudar a otro que está en \\
problemas
\end{tabular}


ITEMS DE LA ESCALA MARLOWE-CROWNE

8. Cuando como en casa, mis modales son tan adecuados como cuando como afuera en un restaurant

9. Si pudiera entrar a un cine sin pagar y estar seguro de no ser visto, probablemente lo haría

28. A veces estuve muy envidioso de la buena suerte de los otros

12. A veces me he rebelado contra personas con autoridad, aunque sabía que estaban en lo cierto

33. Algunas veces me ha molestado que la gente me pida favores

7. Siempre soy cuidadoso acerca de mi manera de vestir

31. Algunas veces pienso, cuando los otros tienen una desgracia, que ellos solo tienen lo que se merecen

29. Casi nunca he sentido el impulso de dejar de lado a alguien

1. Antes de votar averiguo a fondo las capacidades de todos los candidatos

4. Nunca tengo una antipatía intensa hacia alguien

5. En ocasiones, he tenido dudas acerca de mi habilidad para tener éxito en la vida

23. Ha habido ocasiones en las que me sentí como "quebrado"

3. Algunas veces me es difícil continuar con mi trabajo si no estoy estimulado

11. A veces me gusta chusmear

14. Alguna vez "me hice el enfermo" para evitar algo

30. Nunca he sentido que fui castigado sin motivo

32. Nunca he dicho deliberadamente algo que hiera los sentimientos de .21 alguien

6. Algunas veces me siento mal cuando no puedo encontrar mi camino

10. En pocas ocasiones, he dejado de hacer algo debido a que me consideré poco capaz

18. No es particularmente difícil para mí relacionarme con gente "bocona", detestable

21. Siempre soy amable, aún con personas que son desagradables

22. Realmente, a veces insistido en hacer las cosas a mi manera .42

Luego de eliminar los ítems $1,4,7,10,18,28,29,30,31,32$ y 33, se realizó nuevamente el análisis de la estructura interna de la escala (ver Tabla 4) M - C, KMO y Prueba de Bartlett. 
Tabla 4.

Estructura interna de la escala de Marlowe Crowne reducida.

Medida de adecuación muestral de Kaiser-Meyer-Olkin.

Prueba de esfericidad de Bartlett

Chi-cuadrado aproximado

1124.26

Gl

Sig. .000

Se observó que el índice KMO disminuyó a .829 y los factores explicaron un $27.95 \%$ de la varianza. Luego se realizó nuevamente el análisis de componentes principales de la muestra total, tras eliminar los 11 ítems mencionados. Se observó que la estructura bifactorial en esta versión reducida se mantenía (ver Figura 2).

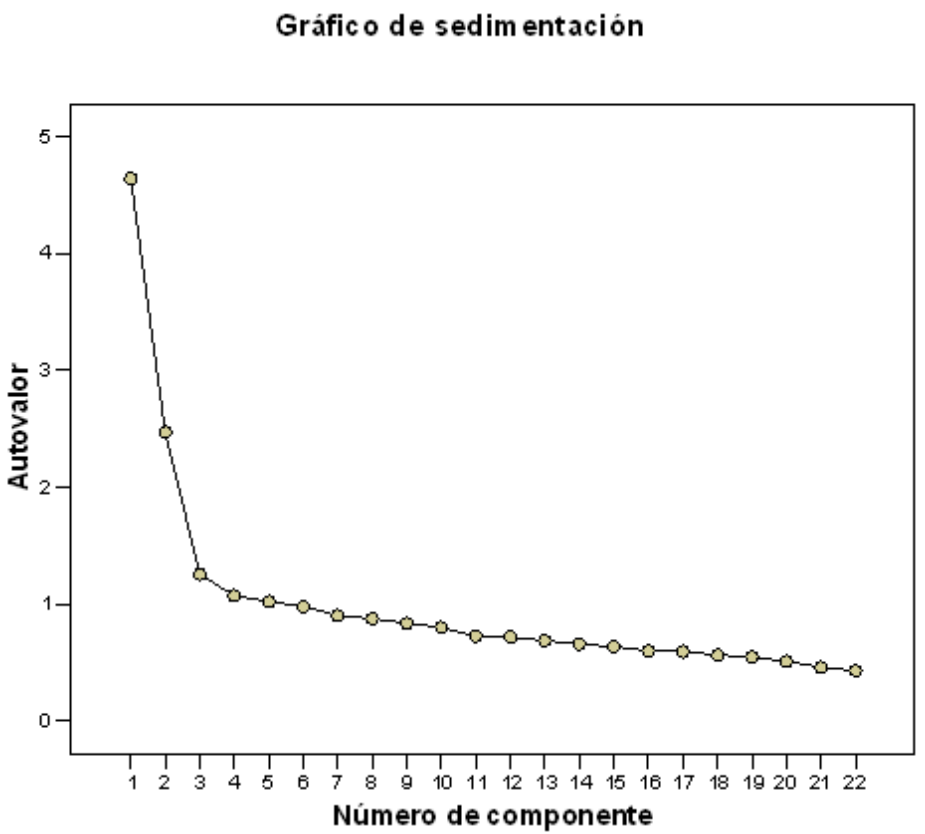

Figura 2. Análisis de componentes principales de la muestra total $(\mathrm{N}=400)$ en la escala $\mathrm{M}-\mathrm{C}$ reducida.

En la tabla 5 se presenta nuevamente la matriz de componentes rotados con el método Varimax, a los fines de observar el aporte factorial de cada ítem por factor de la misma escala, tras eliminar los reactivos señalados. El ítem 23 ("Han habido ocasiones en las que me sentí como quebrado") fue eliminado por considerárselo complejo desde el punto de vista psicométrico. 
Tabla 5.

Matriz de componentes rotados (método Varimax) Versión reducida de la escala $M-C$.

\section{Componente}

ITEMS DE LA ESCALA MARLOWE-CROWNE

1

24. Nunca permitiría que alguien sea castigado por mi culpa .65

20. Cuando no sé algo, no tengo problema en admitirlo .63

13. No importa quien me está hablando, siempre soy un buen escucha

25. Nunca me resiento si me piden que devuelva un favor .58

17. Siempre trato de practicar lo que predico .58

16. Siempre estoy dispuesto a admitir cuando cometo un error .54

19. Algunas veces trato de vengarme más que perdonar y olvidar $-.53$

2. Nunca dudo en dejar mis cosas de lado para ayudar a otro que está en problemas

26. Nunca me he molestado cuando las personas expresan ideas muy diferentes a las mías

22. Realmente, a veces he insistido en hacer cosas a mi manera

27. Nunca hago un viaje largo sin comprobar la seguridad de mi auto

15. En ciertas ocasiones tomé ventajas de alguien

21. Siempre soy amable, aún con personas que son desagradables .45

9. Si pudiera entrar a un cine sin pagar y estar seguro de no ser visto, probablemente lo haría

8. Cuando como en casa, mis modales son tan adecuados como cuando como afuera en un restaurante

12. A veces me he rebelado contra personas con autoridad, aunque sabía que estaban en lo cierto

6. Algunas veces me siento mal cuando no puedo encontrar mi camino

5. En ocasiones, he tenido dudas acerca de mi habilidad para tener éxito en la vida

3. Algunas veces me es difícil continuar con mi trabajo si no estoy estimulado

11. A veces me gusta chusmear

23. Ha habido ocasiones en las que me sentí como quebrado

14. Alguna vez "me hice el enfermo" para evitar algo

Para medir la consistencia interna del instrumento, se utilizó el índice Alfa de Cronbach, que en la escala $\mathrm{M}-\mathrm{C}$ original de 33 ítems fue de .48. Se interpreta que la consistencia interna de la misma fue moderadamente aceptable. Dicho coeficiente se 
incrementó a .57 luego de eliminarse los 12 ítems con baja carga factorial (ítems 1, 4, 7, 10, 18, 23, 28, 29, 30, 31, 32 y 33). También se analizó la fiabilidad para ambos factores extraídos (tras la eliminación de los ítems 1, 4, 7, 10, 18, 23, 28, 29, 30, 31, 32 y 33), los cuales arrojaron un Alfa de Cronbach de .51 (factor 1) y .58 (factor 2). Se les asignó respectivamente los nombres "factor positivo" y "factor negativo" para hacer alusión a aquellos ítems que se refieren a aspectos que tienen que ver con cualidades positivas de autopresentación (factor 1); y aquellos referidos a aspectos que, desde la mirada social, son considerados como negativos (factor 2). Por lo tanto, los dos factores extraídos coincidieron con los que Paulhus (1984) denominó: a) positividad del autoengaño, y b) gestión o manejo de la impresión.

Con el propósito de indagar las características diferenciales de DS en la muestra extraída inicialmente $(\mathrm{N}=400)$, se realizó un análisis correlacional entre el puntaje de la escala total (original) y variables sociodemográficas, tales como edad y nivel educativo. Se obtuvieron las siguientes correlaciones (tabla 6):

- Correlación positiva muy significativa entre la edad de los sujetos y la DS.

- Correlación negativa muy significativa entre la edad y el factor negativo.

- Correlación negativa significativa entre el nivel educativo de los sujetos y las puntuaciones de DS para la escala total (en la versión reducida de la escala tal correlación no aparece).

Tabla 6.

Análisis correlacional entre variables demográficas y puntaje total en la Escala Marlowe Crowne $(N=400)$.

\begin{tabular}{lcccccc}
\hline & & Edad & $\begin{array}{c}\text { Factor } \\
\text { Positivo }\end{array}$ & $\begin{array}{c}\text { Factor } \\
\text { Negativo }\end{array}$ & Educación & $\begin{array}{c}\text { Escala } \\
\text { reducida }\end{array}$ \\
\hline F. Positivo & $\mathrm{R}$ & .04 & & & & \\
& $\mathrm{P}$ & .45 & & & & \\
F. Negativo & $\mathrm{R}$ & $-.27^{* *}$ & .07 & & & \\
Educación & $\mathrm{P}$ & .00 & 16 & & & \\
& $\mathrm{R}$ & -.09 & -.06 & .10 & & \\
Escala & $\mathrm{P}$ & .05 & .23 & .05 & & \\
reducida & $\mathrm{R}$ & -.10 & $.88^{* *}$ & $.54^{* *}$ & -.00 & \\
Escala total & $\mathrm{P}$ & .05 & .00 & .00 & .94 & $.25^{* *}$ \\
& $\mathrm{R}$ & $.27^{* *}$ & $.57^{* *}$ & $-.47^{* *}$ & $-.12^{*}$ & .00 \\
\hline
\end{tabular}

(*) $\mathrm{p}<.01 \quad(* *) \mathrm{p}<.001$ 
A continuación, se analizó la variable sexo aplicando la prueba t de Student (tabla 7). Se observaron diferencias estadísticamente muy significativas entre mujeres y hombres en el nivel educativo $(p=.00)$. También, las medias en el grupo de mujeres fue significativamente mayor $(\mathrm{p}=.00)$ que la de los varones en el factor negativo ("manejo de impresión").

\section{Tabla 7.}

Diferencias de medias de puntajes de DS en hombres y mujeres $(\mathrm{N}=400)$.

\begin{tabular}{|c|c|c|c|c|c|c|}
\hline \multirow{3}{*}{ Educación } & Sexo & $\mathbf{N}$ & Media & DE & $\mathbf{T}$ & $\mathbf{P}$ \\
\hline & Mujeres & 245 & 5.93 & .97 & \multirow[b]{2}{*}{4.29} & \multirow{2}{*}{.00} \\
\hline & Varones & 155 & 5.48 & 1.05 & & \\
\hline \multirow{2}{*}{$\begin{array}{l}\text { Escala M - C } \\
\text { Puntaje Total }\end{array}$} & \multirow{2}{*}{$\begin{array}{l}\text { Mujeres } \\
\text { Varones }\end{array}$} & 245 & 17.98 & 5.19 & \multirow[b]{2}{*}{-1.69} & \multirow[b]{2}{*}{.09} \\
\hline & & 155 & 18.88 & 5.21 & & \\
\hline \multirow[t]{2}{*}{ Positivo } & Mujeres & 245 & 10.07 & 2.36 & \multirow{2}{*}{-.58} & \multirow[b]{2}{*}{.56} \\
\hline & Varones & 155 & 10.22 & 2.64 & & \\
\hline \multirow[t]{2}{*}{ Negativo } & Mujeres & 245 & 3.53 & 1.30 & \multirow{2}{*}{4.74} & \multirow{2}{*}{.00} \\
\hline & Varones & 155 & 2.86 & 1.48 & & \\
\hline \multirow[t]{2}{*}{ Escala reducida } & Mujeres & 245 & 13.59 & 2.78 & \multirow[b]{2}{*}{1.68} & \multirow[b]{2}{*}{.09} \\
\hline & Varones & 155 & 13.08 & 3.13 & & \\
\hline
\end{tabular}

\section{Discusión}

La El análisis de la escala Marlowe Crowne se llevó a cabo con el objetivo de obtener una versión aplicable de la misma para la población de Cuyo (Argentina).

Como se ha mencionado, históricamente el análisis de los instrumentos de respuesta socialmente deseable (RSD) ha revelado dos factores primarios. Paulhus (1984) los denominó: (a) "positividad de autoengaño" (una honesta pero demasiado positiva presentación de sí mismo), y (b) “gestión de la impresión” (auto-presentación adaptada a un público, negando aspectos negativos de sí mismo).

Millham (1974); Ramanaiah y Martin (1980) denominaron a los dos factores hallados como: "factor de atribución" (tendencia a adjudicarse conductas socialmente deseables) y "factor de negación" (tendencia a negar conductas indeseables).

Es de destacar que dichos factores están definidos por los ítems redactados en sentido positivo y negativo en la escala M - C. En el presente trabajo, se confirmó la existencia de tal estructura bifactorial en la escala M - C. 
Puede afirmarse entonces que, en consonancia con investigaciones de la RSD de Borkenau y Ostendorf (1989); Paulhus (1984); Edwards y Walsh (1964); Wiggins (1964); Jackson y Messick (1962) la escala M - C reducida y adaptada a la población cuyana, carga sobre ambos factores aunque, en mayor medida, en la gestión de impresión, es decir en el "factor negativo". (Se eliminaron un total de 12 ítems de la escala original (33 ítems) de los cuales 8 correspondían al factor negativo, 2 al positivo y 2 fueron ítems complejos que arrojaron puntuaciones en ambos factores).

En la escala total se encontró que, a medida que se incrementaba la edad de los sujetos, aumentaba significativamente la tendencia a mostrar una imagen socialmente aceptable de sí mismos ante los demás, rechazando las cualidades individuales consideradas como negativas. No sucedió lo mismo con el nivel educativo, ya que en la población estudiada (mayormente femenina) se observó que, a medida que aumentaba la escolaridad, disminuía la tendencia a mostrar una imagen socialmente aceptable. Esto no apareció en la escala abreviada.

Al estudiar la correlación entre sexo y DS, se halló una asociación muy significativa entre el factor negativo y el sexo femenino, es decir que las mujeres de la población estudiada demostraron mayor tendencia a negar, ante la mirada del otro, características de su personalidad consideradas como negativas o indeseables. Por lo tanto, se infiere que éstas presentaban una mayor necesidad de aprobación social y/o temor al rechazo de los demás que los hombres.

Finalmente, se evalúa que la escala M - C reducida (en función de los resultados de la investigación realizada) constituiría un instrumento moderadamente aceptable para su aplicación en la población cuyana.

\section{Referencias}

Bernreuter, R.G. (1933). Validity of the personality inventory. Personality Journal, 11, 383-386.

Block, J. (1965). The challenge of response sets. New York: Appleton-Century-Crofts.

Borkenau, P., \& Ostendorf, F. (1992). Social desirability scales as moderator and suppressor variables. European Journal of Personality, 6, 199-214.

Crandall, V. C., Crandall, V. J., \& Katkovsky, W. (1965). A children's social desirability questionnaire. Journal of Consulting Psychology, 29, 27-36.

Crowne, D. P. (1979). The experimental study of personality . Hillsdale, NJ: Erlbaum. 
Edwards, A. L. (1957). The social desirability variable in personality assessment and research. New York: Dryden.

Edwards, A. L., \& Walsh, J. A. (1964). Response sets in standard and experimental personality scales. American Educational Research Journal, 1, 52-61.

Eysenck, H. J. \& Eysenck, S. B. G. (1976). Psychoticism as a dimension of personality. New York: Crane, Russak y Company.

Gough, H. G. (1952). On making good impression. Journal of Educational Research, 46, 33-42.

Holden, R. R., (1994). Social Desirability. In R. J. Corsini (ed). Enciclopedia of Psychology (2 ${ }^{\text {nd }}$ ed, vol. 3, pp. 429-430). New York: John Wiley.

Jackson, D. N., \& Messick, S. (1962). Response styles and the assessment of psychopathology. In S. Messick y J. Ross (Eds.), Measurement in personality and cognition (pp. 129-155). New York: Wiley.

Marlowe, D. \& Crowne, D. P. (1960). A new escale of social desirability independent of psychopathology. Journal of Consulting Psychology, 24, 349-354.

Marlowe, D. \& Crowne, D. P (1964). The approval motive: Studies in evaluative dependence. New York: Wiley.

Meehl, P. E. \& Hathaway, S. R. (1946). The K factor as a suppressor variable in the MMPI. Journal of Applied Psychology, 30, 525-564.

Millham, J. (1974). Two components of need for approval score and their relation hip to cheating following success and failure. Journal of Research in Personality, 8, 378-392.

Paulhus, D. L. (1984). Two-component models of socially desirable responding. Journal of Personality and Social Psychology, 46, 598-609.

Pauhlus, D. L. (1986). Self-deception and impression management in test responses. In A Angleitner and J. S. Wiggins (Eds). Personality assessment via questionnaire (pp. 143 - 165). New York: Springer-Verlag.

Ramanaiah, N. V., \& Martin, H. J. (1980). On the two-dimensional nature of the Marlowe-Crowne Social Desirability Scale. Journal of Personality assessment, 44, 504-514.

Reynolds, W. M. (1992). Development of reliable and valid short forms of the Marlowe-Crowne Social Desirability scale. Journal of Clinical Psychology, 38, 119-125.

Schmitt, M. J., \& Steyer, R. (1993). A latent state-trait model (not only) for social desirability. Personality and Individual Differences, 14, 519-529.

Schuessler, K, Hittle, D., \& Cardascia, J. (1978). Measuring responding desirably with attitudeopinion items. Social Psychology, 41, 224-235.

Vernon, P. E. (1934). The attitude of the subject in personality testing. Journal of Applied Psychology, 18, 165-177. 
Wiggins, J. S. (1964). Convergences among stylistic response measures from objective personality. Educational and Psychological Measurement, 24, 551-562. 